\title{
0949 PAN AMERICAN HEALTH ORGANIZATION COLLABORATING CENTRES FOR VIOLENCE AND INJURY PREVENTION
}

E Rodrigues*, A Guedes, P Maurice, M I Gutierrez, M Hijar, S Smith, N Tran, M Ballesteros, M F Tourinho Peres Correspondence: Pan American Health Organization, 525 23rd Street, NW Washington DC, 20037, USA

10.1136/ip.2010.029215.949

Objective Road traffic injuries and violence continue to be important public health problems throughout the Americas. This workshop will describe the role World Health Organization/Pan American Health Organization (WHO/ PAHO) Collaborating Centers (CC) play in supporting WHOs goals for violence and injury prevention in the Americas, particularly in achieving the 2011 strategic objectives for the region.

Materials and Methods CCs form an inter-institutional collaborative network with seven centres in the PAHO region, which work in the area of Violence and Injury Prevention. PAHO has developed a strategic plan that establishes targets for regional expected results (RER) targeting the development of institutional capacity at the national level, development and implementation of national plans for violence prevention and road safety, strengthening of information systems, and conducting cost analysis.

Results In 2009, the PAHO countries had increased their institutional capacity for violence and injury prevention, they had implemented National Plans for violence prevention and road safety, also had established systems for the collection and management of data on violence and road traffic injuries, and they carried out work to understand the cost of injuries. To identify strategies for improving upon these indicators, a meeting of CCs was convened in Sao Paulo in November 2009; targets for 2011 for these indicators were established.

Discussion and Conclusion To reach the 2011 targets and adequately address violence prevention and road safety promotion, countries need to learn from each others successes and work together. CC will continue supporting PAHO to achieve these goals. 